\title{
REGULAR ENDOMORPHISM RINGS OF INJECTIVE MODULES
}

\author{
Sunah Kim* and Soon-Sook Bae
}

\begin{abstract}
The authors have investigated several properties of endomorphism rings of modules. In particular, a closedly simple module and a closedly semi-simple module were defined and then the regularities of its rings of endomorphisms were discussed.
\end{abstract}

\section{Introduction}

Assume that ring $R$ is any associative ring with identity. The ring of all $R$-endomorphisms on a left $R$-module ${ }_{R} M$ (a right $R$-module $M_{R}$ ) is denoted by $\operatorname{End}_{R}(M)$ is studied. In the endomorphism ring $S=\operatorname{End}_{R}(M)$ under the multiplication, i.e., the composition mapping, which is operating on the left side of ${ }_{R} M$. Any left $R$-module ${ }_{R} M$ is considered as a left $R$ - and left $S$-module ${ }_{R-S} M$. Precisely speaking, the composition of endomorphisms is written by the usual order of mappings as usual;

$$
g f: M \underset{f}{\longrightarrow} \longrightarrow \underset{g}{\longrightarrow} M .
$$

We have some materials to recall:

$$
I_{L}=\operatorname{Hom}_{R}(L, 0)=\left\{f \in \operatorname{End}_{R}(M) \mid L \leq \operatorname{ker} f\right\}
$$

is a left ideal of the endomorphism $\operatorname{ring} \operatorname{End}_{R}(M)$, for each submodule $L \leq M$ of $M$

For any subset $J$ of $S=\operatorname{End}_{R}(M)$, let ker $J=\cap_{j \in J} \operatorname{ker} j$ be the intersection of all kernels of endomorphisms in $J$.

Received November 24, 2009. Accepted December 2, 2009.

*: The first author was supported by Chosun University.

2000 Mathematics Subject Classification: 16D10, 16D25, 16D50, 16D60, 16E50,16P40,16S50, $16 \mathrm{~W} 20$.

Keywords and phrases: socle of module, semi-simple, minimal closed, closedly simple, closedly semi-simple, and injective modules. 
We will call $L$ a closed submodule if

$L=\bar{L}$, where $\bar{L}=\cap_{f \in S, L \leq \operatorname{ker} f} \operatorname{ker} f$ is the intersection of all kernels of endomorphisms whose kernels contain $L$.

Recall a module is simple, [3](irreducible, [7]) it has no non-trivial submodule. A module $M$ is said to be semi-simple (completely reducible) if it is a sum of (possibly infinitely many) simple modules.

A submodule $N$ of $M$ is said to be minimal (minimal closed, resp.) if $0 \leq H \leq N$ for any (closed, resp.) submodule $H \leq M$, implies that $0=H$ or $H=N$.

The Socle of a module $M$, denoted by $\operatorname{Soc}(M)$, is the sum of all minimal submodules $N_{\alpha}$ of $M$, in fact,

$$
\operatorname{Soc}(M)=\sum N_{\alpha}, \text { for all minimal submodules } N_{\alpha} \leq M .
$$

Here we see the rhetorical question, which means, that a minimal submodule is simple in the submodule itself, and vice versa.

Definition 1.1. For any left (left) $R$-module $M$,

(1) $M$ is said to be closedly simple if there is no proper closed submodule of $M$ i.e., only closed submodules are 0 and $M$ itself [8].

(2) Let $\left(M_{\alpha}\right)_{\alpha \in \Gamma}$ be an indexed set of left (right) $R$-modules $M_{\alpha}(\alpha \in \Gamma)$. Then a direct product $\prod_{\Gamma} M_{\alpha}$ and a weak direct product $\prod_{\Gamma}^{w} M_{\alpha}$ of $R$-modules $\left(M_{\alpha}\right)_{\alpha \in \Gamma}$ are defined, briefly, saying

$$
\prod_{\Gamma} M_{\alpha}=\left\{\left(m_{\alpha}\right)_{\alpha} \mid m_{\alpha} \in M_{\alpha}(\alpha \in \Gamma)\right\}
$$

under the coordinatewise addition and scalar multiplication;

$$
\left(n_{\alpha}\right)_{\alpha}+\left(m_{\alpha}\right)_{\alpha}=\left(n_{\alpha}+m_{\alpha}\right)_{\alpha} \text { and } r\left(n_{\alpha}\right)_{\alpha}=\left(r n_{\alpha}\right)_{\alpha},
$$

for every $\left(n_{\alpha}\right)_{\alpha},\left(m_{\alpha}\right)_{\alpha} \in \prod_{\Gamma} M_{\alpha}$, and for every $r \in R$.

And $\prod_{\Gamma}^{w} M_{\alpha}=\left\{\left(m_{\alpha}\right) \in \prod_{\Gamma} M_{\alpha} \mid m_{\beta}=0\right.$ for all but a finite number of indices $\left.\beta \in \Gamma\right\}$.

With the $\alpha$-th projection $\pi_{\alpha}: \prod_{\Gamma} M_{\alpha} \rightarrow M_{\alpha}$ defined by $\pi_{\alpha}\left(\left(m_{\alpha}\right)_{\Gamma}\right)=m_{\alpha},(\alpha \in \Gamma)$ we have that $\quad\left(\prod_{\Gamma} M_{\alpha},\left(\pi_{\alpha}\right)_{\Gamma}\right)$ is a direct product of $\left(M_{\alpha}\right)_{\alpha \in \Gamma} \quad$ p.79 [3]. 
Moreover it is clearly known that any weak direct product of $R$-modules is a submodule of a direct product of $R$-modules.

The following definition is widely known.

Definition 1.2. A left $R$-module $E$ is injective in case $E$ is injective relative to every left $R$-module $M$. That is, $E$ is injective in case whenever there is given the solid part of a diagram:

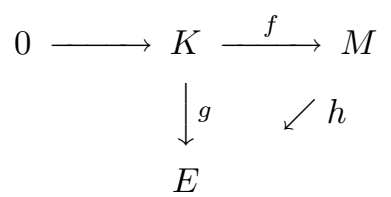

in the category ${ }_{R} \mathcal{M}$ of left $R$-modules with row exact, there is an

$R$-homomorphism $h$ such that the whole diagram commutative; $h f=g$. In other words, the injective modules are the arrow-theoretic or categorical duals of the projective modules.

A left module ${ }_{R} M$ is said to be quasi-injective in case it is $M$-injective (injective relative to $M$ ).

It is well-known that every injective module is quasi-injective.

Recall that 16.11 Corollary (p.186, [3]): for any indexed set $\left(E_{\alpha}\right)_{\alpha \in \Gamma}$ of left $R$-modules, we have $\prod_{\Gamma} E_{\alpha}$ is injective if and only if each $E_{\alpha}$ is injective.

The following proposition is 18.8. Corolllary on p.206, [3].

Proposition 1.3. A ring $R$ is semi-simple if and only if every left $R$-module is injective.

Of course, another fact about semi-simple ring is found in [6]. If $R$ is semisimple, then every module is semi-simple. However the converse does not hold, in general. For instance, $\mathbb{Z}_{p} \oplus \mathbb{Z}_{p}$ (modulo $p$ ) is a semi-simple $\mathbb{Z}$-module, but the integer ring $\mathbb{Z}$ is not semi-simple.

Definition 1.4. For a commutative ring $R$ with identity, a left (right, resp.) $R$ - module ${ }_{R} M$ ( $M_{R}$, resp.) is said to be a multiplication module if each submodule $K \leq M$, there is an ideal $I$ of $R$ such that $K=I M$ ( $M I$, resp.).

The following properties of multiplication modules are found from [10].

Proposition 1.5. For a commutative ring $R$ with identity, $R$ is semi-simple if and only if every multiplication module is semi-simple. 
Lemma 1.6. For any commutative ring $R$ with identity and for any multiplication left (right, resp.) $R$-module ${ }_{R} M$ ( $M_{R}$ resp.), if $I$ is any minimal ideal of $R$, then we have $I M(M I$, resp.) is a minimal submodule of $M$ or $I M=0(M I=0$, resp. $)$.

Proposition 1.7. For any commutative ring $R$ with identity, the following are equivalent:

(1) $R$ is semi-simple;

(2) $R$ is a direct sum of simple rings;

(3) Every multiplication module ${ }_{R} M$ is semi-simple;

(4) Every multiplication module ${ }_{R} M$ is a direct sum of simple modules.

Recall that an associative ring $R$ with identity is said to be regular if $R$ is von-Neumann regular, that is, for any $a \in R$, there is $b \in R$ such that $a=a b a$.

\section{Results}

To study the regular endomorphism rings of modules, we need to develope the properties of closed submodules and minimal closed submodules of left (right) $R$-modules.

Proposition 2.1. For any left (right) $R$-module $M$, we have the following:

(1) For sumodule $K \leq M$, we have $K \leq \bar{K}$.

(2) For submodules $K, L \leq M$, we have

$$
\overline{K \cap L} \leq \bar{K} \cap \bar{L}
$$

and

$$
\overline{K+L}=\overline{\bar{K}+\bar{L}} .
$$

(3) If $N \leq M$ is minimal, then $\bar{N}$ is a minimal closed submodule of $M$.

(4) The converse of the item (3) does not hold, in general.

(5) For each pair of minimal submodules $N_{1}, N_{2} \leq M$ we have

$$
N_{1} \cap N_{2}=\left\{\begin{array}{l}
0, \\
N_{i}(i=1,2) .
\end{array}\right.
$$


(6) For each pair of minimal closed submodules $N_{1}, N_{2} \leq M$ we have

$$
\overline{N_{1} \cap N_{2}}=\left\{\begin{array}{l}
0, \\
\overline{N_{i}}=N_{i}(i=1,2) .
\end{array}\right.
$$

Proof. (1): It is elementary.

(2): $\bar{K} \cap L \leq \bar{K} \cap \bar{L}$ is obvious.

Comparing left ideals $I_{K+L}=I_{K} \cap I_{L}$ with submodules $K$ and $L$, we can see that their kernels are the same. So, $\operatorname{ker} I_{K+L}=\overline{K+L}=\operatorname{ker}\left(I_{K} \cap I_{L}\right)=\overline{\bar{K}}+\bar{L}$.

(3): If $K$ is any closed submodule of $M$ such that $0 \leq K=\bar{K} \leq \bar{N}$. Then by the computation of sets; $\bar{N} \cap \bar{K}=\bar{N} \cap K=K \geq \overline{N \cap K}$. If $N \cap K=0$, then since $N$ is minimal we have $K=0$. And if $N \cap K \neq 0$, then since $N$ is minimal, we have $N \leq K$. Thus $\bar{N} \leq \bar{K}=K$. Then $\bar{N}=\bar{K}=K$. Hence $\bar{N}$ is a minimal closed submodule of $M$, for any minimal submodule $N$ of $M$.

(4): Take the ring $\mathbb{Z}$ of all integers and the left $\mathbb{Z}$-module $\mathbb{Z} \mathbb{Q}$ regarded as an additive group $\mathbb{Q}$, by the Generalized Schur's Lemma II with an injective closely simple module $\mathbb{Q}$ we have a division ring $E n d_{\mathbb{Z}}(\mathbb{Q}$ ) ( with keeping in mind, $E n d_{\mathbb{Q}}(\mathbb{Q})$ is a division ring by the Schur's Lemma), thence $\overline{k \mathbb{Z}}=\mathbb{Q}$ is a minimal closed submodule of $\mathbb{Q}$, however $k \mathbb{Z}$ (for any natural number $k \in \mathbb{N}$ ) is not minimal since $k \mathbb{Z} \leq \mathbb{Q}$, of course, $\mathbb{Z}$ is not Artinian [5].

(5) and (6) are proved by applying (3).

From the property of (6) of Proposition 2.1 we can make a new definition of a module.

Definition 2.2. A module $M$ is said to be closedly semi-simple if it is a sum of closedly simple submodules of $M$.

Note 2.3. For any semi-simple module $M$,

$$
M=\operatorname{Soc}(M)=\sum N_{\alpha}=\oplus N_{\alpha} \text { for all minimal submodules } N_{\alpha} \text { of } M .
$$

And also for any closedly semi-simple module $M$,

$$
\begin{aligned}
M & =\sum \overline{N_{\alpha}}=\oplus \overline{N_{\alpha}} \\
& =\left\{\sum_{\text {finite }} n_{\alpha} \mid n_{\alpha} \in \overline{N_{\alpha}}, n_{\beta}=0, \text { for all but a finite number of indices } \beta \in \Gamma\right\} \\
& =\left\{\oplus n_{\alpha} \mid n_{\alpha} \in \overline{N_{\alpha}}, n_{\beta}=0, \text { for all but a finite number of indices } \beta \in \Gamma\right\},
\end{aligned}
$$

for all minimal closed submodules $\overline{N_{\alpha}}$ of $M$ (See (5) and (6) of Proposition 2.1). 
REMARK 2.4. Every semi-simple module is always closedly semi-simple but the converse is not true, in general. The Abelian groups $\mathbb{Z} \oplus_{1}^{\infty} \mathbb{Q}$ ( or $\oplus^{n} \mathbb{Q}(n \in$ $\mathbb{N})$ ) is not semi-simple but closedly semi-simple $\mathbb{Z}$-module.

Usually we will ignore the trivial case of $M=0 \oplus M=M+M$.

LEmma 2.5. For any left (right) $R$-module $M$, the following are equivalent

(1) $M$ is closedly simple;

(2) Any non-zero endomorphism is a monomorphism.

Proof. For each $f \in \operatorname{End}(M)$, we have a closed submodule $\operatorname{ker} f$ of $M$, thus ker $f=0$ if and only if $f$ is a monomorphism. Hence the proof is completed.

Proposition 2.6. For any (quasi-)injective left (right) $R$-module $M$, if $M$ is closedly simple, then $f: M \rightarrow M$ is left invertible, for any non-zero endomorphism $f$.

Proof. From Lemma 1.4 because of the (quasi-)injectivity of $M$ the following commutative diagram aids to complete the proof:

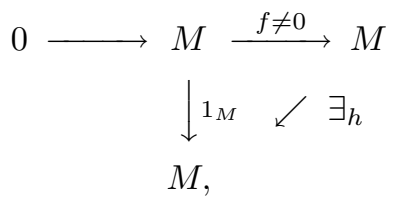

where $1_{M}: M \rightarrow M$ is the identity mapping on $M$. Thus for any non-zero endomorphism $f: M \rightarrow M$ is a monomorphism, since $M$ is injective there exists an endomorphism $h: M \rightarrow M$ such that $1_{M}=h f$, where $1_{M}: M \rightarrow M$ is the identity mapping on $M$. Thus $f$ is left invertible.

Lemma 2.7. For any closedly simple module $M$, if $M=\sum_{\alpha \in \Gamma} N_{\alpha}$, for all closedly simple submodules $N_{\alpha}(\alpha \in \Gamma)$ of $M$, then $\oplus_{\Gamma} N_{\alpha}$ and $\prod_{\Gamma}^{w} N_{\alpha}$ are isomorphic, simply denoted by $M=\oplus_{\Gamma} N_{\alpha} \simeq \prod_{\Gamma}^{w} N_{\alpha} \leq\left(\prod_{\Gamma} N_{\alpha}\right)$.

Proof. Since each closedly simple submodule $N_{\alpha}(\alpha \in \Gamma)$ is a minimal closed submodule of $M$, by (6) of Proposition 2.1, we have that $\sum_{\Gamma} N_{\alpha}=\oplus N_{\alpha}$. Define $\phi: \oplus_{\Gamma} N_{\alpha} \rightarrow \prod_{\Gamma}^{w} N_{\alpha}$ by $\oplus_{\Gamma} n_{\alpha} \mapsto\left(n_{\alpha}\right)_{\alpha \in \Gamma}$. Then it is easily proved $\phi$ is a welldefined, $R$-homomorphism, monomorphism, and epimorphism. Therefore $\phi$ is an $R$-isomorphism. 
Lemma 2.8. If a left (right) $R$-module $M$ is closedly semi-simple, then ker $f$ is a direct summand of $M$, for any non-zero non-monomorphism $f$ in $\operatorname{End}(M)$.

Proof. Let $f \in \operatorname{End}_{R}(M)$ be not a monomorphism. Then clearly ker $f \neq 0$. $\operatorname{ker} f \lesseqgtr M$ by $f \neq 0$ and $\operatorname{ker} f \cap M=\operatorname{ker} f \cap\left(\sum N_{\alpha}\right)$, where $M=\sum N_{\alpha}$, for all minimal closed submodules $N_{\alpha}(\alpha \in \Gamma)$, for some index set $\Gamma$. Now then we partition the index set $\Gamma$ into two disjoint sets $A$ and $B$ such that

$$
A=\left\{\alpha \in \Gamma \mid \operatorname{ker} f \cap N_{\alpha} \neq 0\right\} \text { and } B=\left\{\alpha \in \Gamma \mid \operatorname{ker} f \cap N_{\alpha}=0\right\} .
$$

Notice here ker $f \cap N_{\alpha}(\neq 0)$ is a closed submodule contained in $N_{\alpha}$, we have $N_{\alpha} \leq \operatorname{ker} f$, for each $\alpha \in A$.

From the following computations

$$
M=\sum_{\Gamma} N_{\alpha}=\sum_{A} N_{\alpha} \oplus \sum_{B} N_{\beta} \leq \operatorname{ker} f \oplus \sum_{B} N_{\beta}
$$

and $N_{\alpha} \leq \operatorname{ker} f$ for any $\alpha \in A$ by the minimal closed submodule $N_{\alpha}$, it follows that $\sum_{A} N_{\alpha}=\operatorname{ker} f$, being a direct summand of $M$.

Applying 20.2 Proposition in [3] as functors direct sum $\oplus$ and direct product $\prod$ from the category ${ }_{R} \mathcal{M}$ of left $R$-modules to the category $\mathbb{Z} \mathcal{M}$ of left $\mathbb{Z}$-modules:

(1) $\operatorname{Hom}_{R}\left(\oplus_{\Gamma} U_{\alpha},-\right) \simeq \prod_{\Gamma} \operatorname{HomR}\left(U_{\alpha},-\right)$;

(2) $\operatorname{Hom}_{R}\left(-, \prod_{\Gamma} U_{\alpha}\right) \simeq \prod_{\Gamma} \operatorname{Hom}_{R}\left(-, U_{\alpha}\right)$.

We recall the following properties of direct product and weak direct product modules and the Hom functor are followed practically;

(1) If $B \simeq C$ are isomorphic $R$-modules, then $\operatorname{Hom}(B, A) \simeq \operatorname{Hom}(C, A)$ and $\operatorname{Hom}(A, B) \simeq \operatorname{Hom}(A, C)$, for every $R$-module $A$.

(2) For any indexed set $\left(M_{\alpha}\right)_{\alpha \in \Gamma}$ of left (right) $R$-modules $M_{\alpha}(\alpha \in \Gamma)$, then

$$
\operatorname{Hom}\left(\prod_{\Gamma}^{w} M_{\alpha}, \prod_{\Gamma}^{w} M_{\alpha}\right) \simeq \operatorname{Hom}\left(\prod_{\Gamma} M_{\alpha}, \prod_{\Gamma} M_{\alpha}\right) .
$$

(Consider the mapping

$$
\operatorname{Hom}\left(\prod_{\Gamma} M_{\alpha}, \prod_{\Gamma} M_{\alpha}\right) \rightarrow H o m\left(\prod_{\Gamma}^{w} M_{\alpha}, \prod_{\Gamma}^{w} M_{\alpha}\right):\left.f \mapsto f\right|_{\prod_{\Gamma}^{w} M_{\alpha}},
$$


where $\left.f\right|_{\prod_{\Gamma}^{w} M_{\alpha}}$ is the restriction of $f$ to the submodule $\prod_{\Gamma}^{w} M_{\alpha}$ of $\prod_{\Gamma} M_{\alpha}$, for each $f \in \operatorname{Hom}\left(\prod_{\Gamma} M_{\alpha}, \prod_{\Gamma} M_{\alpha}\right)$, which is required.)

(3) For any closedly semi-simple module $M=\sum_{\Gamma} N_{\alpha}$, for all minimal closed submodules $N_{\alpha}$ of $M$, it is concluded immediately:

$$
\begin{aligned}
\operatorname{Hom}\left(N_{\alpha}, \prod_{\Gamma}^{w} N_{\alpha}\right) & \simeq \operatorname{Hom}\left(N_{\alpha}, \prod_{\Gamma} N_{\alpha}\right) \simeq \prod_{\beta \in \Gamma} \operatorname{Hom}\left(N_{\alpha}, N_{\beta}\right) . \\
\operatorname{End}(M)=\operatorname{End}\left(\oplus_{\Gamma} N_{\alpha}\right) & =\operatorname{Hom}\left(\oplus_{\Gamma} N_{\alpha}, \oplus_{\Gamma} N_{\beta}\right) \simeq \operatorname{Hom}\left(\oplus_{\Gamma} N_{\alpha}, \prod_{\Gamma}^{w} N_{\beta}\right) \\
& \left.\simeq \prod_{\Gamma} \operatorname{Hom}\left(N_{\alpha}, \prod_{\Gamma}^{w} N_{\beta}\right)=\prod_{\Gamma} \operatorname{Hom}\left(N_{\alpha}, \prod_{\Gamma} N_{\beta}\right)\right) \\
& \simeq \prod_{\Gamma \times \Gamma} \operatorname{Hom}\left(N_{\alpha}, N_{\beta}\right) .
\end{aligned}
$$

Theorem 2.9. For any (quasi-)injective module $M$, if $M$ is closedly semisimple, then $\operatorname{End}_{R}(M)$ is regular.

Proof. Since on every (quasi-)injective module $M$ every monomorphism $f$ is left invertible. So $f$ has a left inverse $g$ in $\operatorname{End}(\mathrm{M})$, and thus $f=f g f$, for some $g \in \operatorname{End}(M)$. So the regularity of any monomorphism on $M$ is obtained immediately.

The reglarity of any non-zero non-monomorphism $f: M=\oplus N_{\alpha} \simeq \prod^{w} N_{\alpha} \rightarrow$ $M=\oplus N_{\alpha}$ is only to be proved. By Lemma 2.8 the kernel ker $f$ is a direct summand of $M$.

Considering the following commutative diagram with chasing element assign:

$$
N_{\alpha}
$$

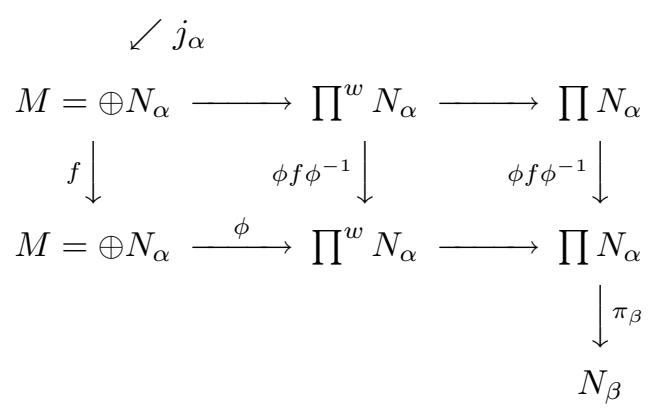




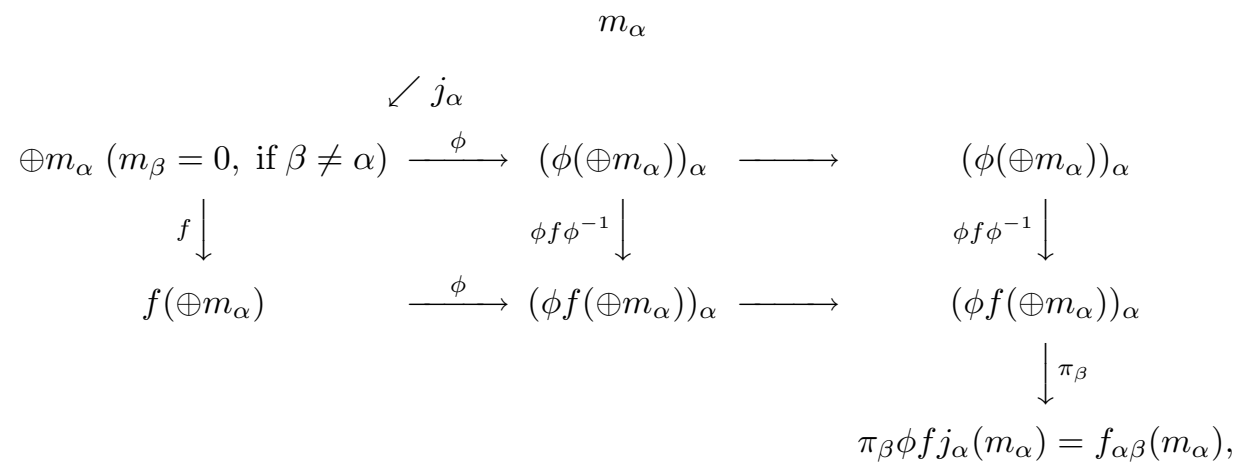

where $j_{\alpha}: N_{\alpha} \rightarrow \oplus_{\Gamma} N_{\alpha}$ is the injection, $\pi_{\beta}: \prod_{\Gamma} N_{\alpha} \rightarrow N_{\beta}$ is the $\beta$-th projection, and $\phi: \oplus_{\Gamma} N_{\alpha} \rightarrow \prod_{\Gamma}^{w} N_{\alpha}$ is the $R$-isomorphism. Let $\pi_{\beta} \phi f j_{\alpha}=$ $f_{\alpha \beta}$. Comparing the kernel of $f$ with the kernel of $f_{\alpha \beta}$, we obtain that $\operatorname{ker} f=$ $\oplus_{\alpha \in \Gamma} \operatorname{ker} f_{\alpha \beta}$ by [3]. Since $f \neq 0$ if and only if there are $\alpha, \beta \in \Gamma$ such that $f_{\alpha \beta} \neq 0$. Thus ker $f_{\alpha \beta}=0$, for some $(\alpha, \beta) \in \Gamma \times \Gamma$ (otherwise, it induces that $f=0$ from $\operatorname{ker} f \geq N_{\alpha}$, for all $\alpha$ ).

On the other hand, define a function $g_{\beta \alpha}=f_{\alpha \beta}^{\leftarrow}: \operatorname{Im} f_{\alpha \beta} \rightarrow M$ (pullback of $f_{\alpha \beta}$ ) defined by the reversing assignment, i.e., $f_{\alpha \beta}\left(n_{\alpha}\right) \mapsto n_{\alpha}$ is an $R$-monomorphism from the $R$-submodule $\operatorname{Im} f_{\alpha \beta}\left(\leq N_{\beta}\right)$ onto the preimage $N_{\alpha}$ (which is possible since $f_{\alpha \beta}: N_{\alpha} \rightarrow N_{\beta}$ is a monomorphism).

Precisely speaking, let $A^{\prime}=\operatorname{ker} f, B^{\prime}=\sum_{\Gamma \backslash\{\alpha\}} N_{\gamma}, C=\sum_{\Gamma \backslash\{\alpha, \beta\}} N_{\delta}$, and $D=\sum_{\Gamma \backslash\{\beta\}} N_{\delta}$. Then we have the following:

$$
\begin{array}{r}
1_{A^{\prime}} \oplus f_{\alpha \beta} \oplus g_{\beta \alpha} \oplus 1_{C}: A^{\prime} \oplus N_{\alpha} \oplus \operatorname{Im} f_{\alpha \beta} \oplus C \stackrel{\leq}{\longrightarrow} \oplus N_{\beta} \oplus N_{\alpha} \oplus C \\
\| \\
A^{\prime} \oplus N_{\beta} \oplus D \\
\| \\
A^{\prime} \oplus B^{\prime} \\
\| \\
\oplus_{\Gamma} N_{\alpha}=M
\end{array}
$$

is the injective inclusion, since $M$ is (quasi-)injective, there is an extension $h: M \rightarrow M$ such that the restriction $\left.h\right|_{A^{\prime} \oplus N_{\alpha} \oplus \operatorname{Im} f_{\alpha \beta} \oplus D}=1_{A^{\prime}} \oplus g_{\beta \alpha} \oplus 1_{D}$ of $h$ to the submodule $A^{\prime} \oplus \operatorname{Im} f_{\alpha \beta} \oplus N_{\beta} \oplus D$. 
Take $h=\left(h_{\gamma \delta}\right): M=\oplus N_{\alpha} \simeq \prod^{w} N_{\alpha} \rightarrow M=\oplus N_{\alpha} \simeq \prod^{w} N_{\alpha}$. Then $h_{\beta \alpha} f_{\alpha \beta}=1_{N_{\alpha}}$, for every $(\alpha, \beta) \in \Gamma \times \Gamma$ follows from the composition $h f=1_{B^{\prime}}$ of $h$ and $f$.

Consider that a direct sum $f: M=A^{\prime} \oplus B^{\prime} \rightarrow M=A^{\prime} \oplus B^{\prime}$ has a left inverse $h \in \operatorname{End}(M)$. Then $f=f h f$ follows.

Therefore the endomorphism ring $\operatorname{End}(M)$ is regular. The proof is completed.

Corollary 2.10. For any (quasi-)injective $R$-module $M$, if $M$ is semisimple, then $\operatorname{End}_{R}(M)$ is regular.

Proof. Since every semi-simple module $M$ is closedly semi-simple the proof is an easy consequence of the above Theorem 2.9.

Recall that a ring $R$ is semi-simple if and only if every $R$-module is injective $([3])$.

Corollary 2.11. Let $R$ be a semi-simple ring. Then $\operatorname{End}(M)$ is regular, for every left (right) $R$-module $M$.

Proof. By Corollary 2.6 p.274 [6], every $R$-module is semi-simple and thus every $R$-module is closedly semi-simple. On the other hand, $R$-modules is injective by Proposition 1.3. The hypotheses of Theorem 2.9 are satisfied. It follows that $\operatorname{End}(M)$ is regular.

Corollary 2.12. If a commutative ring $R$ with identity is semi-simple, then $\operatorname{End}_{R}(M)$ is regular, for any multiplication module $M$ over $R$.

Proof. Applying 18.8. Corollary on p. 206 in [3] to a commutative ring $R$, every left (right) $R$-module is injective. And by Proposition 1.7 every multiplication module $M$ is semi-simple. Thus the hypotheses of Theorem 2.9 are satisfied. Hence $\operatorname{End}(M)$ is regular, for every multiplication module $M$.

Proposition 2.13. If a module $M$ is closedly semi-simple, then the following are equivalent:

(1) $\operatorname{End}(M)$ is regular;

(2) Im $f$ is a direct summand of $M$, for any non-zero $f \in \operatorname{End}(M)$. 
Proof. (1) $\Longrightarrow(2)$ : Automatically it is proved by Corollary 3.2, [2].

$(2) \Longrightarrow(1)$ : By Corollary 3.2 [2], the fact of a direct summand ker $f$ of $M$ follows from the closedly semi-simple module $M$, for every endomorphism $f: M \rightarrow M$ by Lemma 2.8. Together with the hypothesis of a direct summand $\operatorname{Im} f$ of $M$, immediately it follows that $\operatorname{End}(M)$ is regular.

Corollary 2.14. If a module $M$ is semi-simple, then the following are equivalent:

(1) $\operatorname{End}(M)$ is regular;

(2) Imf of $f$ is a direct summand of $M$, for any non-zero $f \in \operatorname{End}(M)$.

Proof. Since each semi-simple module is a closedly semi-simple module the proof is completed by the Proposition 2.13 .

\section{References}

1. I.N.Herstein, Noncommutative rings, The Math. Assoc. of America, 1968

2. Roger Ware, Endomorphism Rings of Projective Modules, Trans. of the AMS 55 (1971), $233-256$.

3. Frank W. Anderson and Kent R.Fuller, Rings and Categories of Modules, New York ; Spring -Verlag, 1973.

4. C. Faith, Rings, Modules, and Categories, Berlin, Springer-Verlag, 1973.

5. Thomas W. Hungerford, Algebra, Spring-Verlag; New York, 1974.

6. Maurice Auslander \& David A. Buchsbaum, Groups, Rings, Modules (1974), Harper \& Row, Publishers, New York, Evanston, San Francisco, London.

7. Joachim Lambek, Lecture on Rings and Modules, Chelsea Publ. Comp. New York, 1976.

8. Soon-Sook Bae, Generalized Schur's Lemmas, Journal of Graduate School, Kyungnam University 16 No.2 (2001), 7-11.

9. A.G. Naoum and W.K.H. Al-Aubaidy, A note on Multiplication Modules and their Rings of Endomorphisms, Kyungpook Math.J. 35 (1995), 223-228..

10. Soon-Sook Bae, On the semi-primitive and the semi-simple Multiplication Modules, Kyungnam University, Journal of Graduate School 11 (1996), 391-397.

11. Carl Faith, Indecomposable injective modules and a theorem of Kaplansky, Communications in algebra 30 (2002), 5875-5889. 
Department of Mathematics,

College of Natural Sciences,

Chosun University, 375 Seosuk-Dong, Gwangju,

501-759, Republic of Korea

E-mail: sakim@chosun.ac.kr

Hyundai Apartment 203-1403,

Wolyoung-Dong 705-2, Masan, Kyungnam,

631-260, Republic of Korea.

E-mail: ssbae2000@msn.com 